\title{
Observational constraints on the nucleosynthesis in the more massive AGB stars
}

\author{
D. A. García-Hernández ${ }^{* \dagger}$ \\ Instituto de Astrofísica de Canarias (IAC), E-38200 La Laguna, Tenerife, Spain \\ Departamento de Astrofísica, Universidad de La Laguna (ULL), E-38206 La Laguna, Tenerife, \\ Spain \\ E-mail: agarciadiac.es
}

\begin{abstract}
Most of the stars $\left(\mathrm{M}<8\right.$ solar mass, $\mathrm{M}_{\text {sun }}$ ) in the Universe end their lives with a phase of strong mass loss and experience thermal pulses on the Asymptotic Giant Branch (AGB). They are one of the main contributors to the enrichment of the interstellar medium and thus to the chemical evolution of galaxies. More specifically, the more massive $\left(\mathrm{M}>4 \mathrm{M}_{\text {sun }}\right)$ AGB stars are expected to form very different isotopes (such as ${ }^{87} \mathrm{Rb},{ }^{7} \mathrm{Li},{ }^{14} \mathrm{~N},{ }^{13} \mathrm{C},{ }^{41} \mathrm{Ca},{ }^{60} \mathrm{Fe},{ }^{26} \mathrm{Al}$ ) from the isotopes formed by lower mass AGB stars and Supernova explosions, as a consequence of different dominant nuclear reaction mechanisms. The discovery that the more massive AGB stars are sources of isotopes (like the long-lived radioisotope ${ }^{87} \mathrm{Rb}$ ) that are not produced in lower-mass stars, confirmed for the first time that these stars produce heavy neutron-rich (s-process) elements via ${ }^{22} \mathrm{Ne}$ as neutron source $[1,2]$. The extreme $\mathrm{Rb}$ abundances and extraordinarily high [Rb/Zr] ratios observed, however, represent a challenge for theoretical AGB nucleosynthesis models $[3,4]$. Very recently, we have used more realistic model atmospheres for massive AGBs that include a gaseous circumstellar envelope [5]. The new $\mathrm{Rb}$ abundances and [Rb/Zr] ratios derived with these dynamical models significantly resolve the problem of the present mismatch between the observations of the more massive Rb-rich AGB stars and the theoretical predictions [5], providing fundamental constraints to the AGB nucleosynthesis models and nuclear physics.
\end{abstract}

XIII Nuclei in the Cosmos,

7-11 July, 2014

Debrecen, Hungary

\footnotetext{
*Speaker.

$\dagger$ D.A.G.H. acknowledges support provided by the Spanish Ministry of Economy and Competitiveness under grant AYA-2011-27754.
} 


\section{AGB stellar nucleosynthesis}

The asymptotic giant branch (AGB) is the last nuclear-burning phase of low- and intermediatemass stars (the majority of the stars in the Universe with $0.8 \leq \mathrm{M} \leq 8$ solar mass, $\mathrm{M}_{\text {sun }}$ ). AGB stars are among the main contributors to the chemical enrichment of the interstellar medium since they suffer strong mass loss together with nucleosynthesis processes. Low-mass AGB stars (M $<4 \mathrm{M}_{\text {sun }}$ ) can turn C-rich (i.e., $\mathrm{C} / \mathrm{O}>1$ ) due to the dredge-up of carbon from the bottom of the convective envelope to the stellar surface. The $s$-process allows the production of elements heavier than iron by slow neutron-captures. In these stars, the ${ }^{13} \mathrm{C}(\alpha, \mathrm{n}){ }^{16} \mathrm{O}$ reaction is assumed to operate as the main neutron source [6,7]. On the other hand, intermediate-mass AGB stars $(4 \leq \mathrm{M} \leq 8$ $\left.\mathrm{M}_{\text {sun }}\right)$ are O-rich stars $(\mathrm{C} / \mathrm{O}<1)$ because of the operation of hot bottom burning (HBB) [8], which burns carbon at the base of the convective envelope, thus preventing the formation of a carbon star. In the latter stars, the s-process elements are expected to mainly form by the neutrons released by the ${ }^{22} \mathrm{Ne}(\alpha, \mathrm{n})^{25} \mathrm{Mg}$ reaction, in a higher neutron density environment than in lower mass AGB stars [1]. The relative abundance of s-elements as $\mathrm{Rb}$ to other neighboring ones such as $\mathrm{Sr}$, $\mathrm{Y}$ and $\mathrm{Zr}$ is an indicator of the neutron density, namely a discriminant of the stellar mass and the main neutron source at the $s$-process site $[1,6,7]$.

In short, the more massive AGB stars form very different isotopes and radionuclides (such as ${ }^{87} \mathrm{Rb},{ }^{7} \mathrm{Li},{ }^{14} \mathrm{~N},{ }^{13} \mathrm{C},{ }^{41} \mathrm{Ca},{ }^{60} \mathrm{Fe},{ }^{26} \mathrm{Al}$ ) from the isotopes formed by lower mass AGB stars and Supernova explosions as the consequence of HBB coupled with s-process nucleosynthesis activated via the ${ }^{22} \mathrm{Ne}$ neutron source [9].

\section{The Rb problem}

Observationally, a low $[\mathrm{Rb} / \mathrm{Zr}]$ ratio $(<0)$ is found in low-mass $\left(<3 \mathrm{M}_{\text {sun }}\right)$ AGB stars [6,7] while higher mass AGB stars display $[\mathrm{Rb} / \mathrm{Zr}]>0$ [1,2,8]. García-Hernández et al. [1,2] (hereafter Paper I and Paper II, respectively) derived the $\mathrm{Rb}$ and $\mathrm{Zr}$ abundances in several Galactic and Magellanic Cloud (MC) intermediate-mass AGB stars among a sample of OH/IR stars; luminous O-rich AGB stars that are extremely bright in the infrared, showing a characteristic double-peaked $\mathrm{OH}$ maser emission at $1612 \mathrm{MHz}$ [8]. The $\mathrm{Rb}$ abundances and [ $\mathrm{Rb} / \mathrm{Zr}]$ ratios found in these objects represent a challenge for theoretical AGB nucleosynthesis models $[3,4]$, which do not predict the extreme $\mathrm{Rb}$ overabundances $([\mathrm{Rb} / \mathrm{Fe}] \geq 2 \mathrm{dex}$ ) and extraordinarily high $[\mathrm{Rb} / \mathrm{Zr}]$ ratios. However, the $\mathrm{Rb}$ abundance was derived from the resonant $\mathrm{Rb}$ I absorption line at $7800 \AA$, using hydrostatic model atmospheres. The $\mathrm{Rb}$ I line is probably affected by contamination from one or more circumstellar (CS) components, as has already been suggested by the detection of blue-shifted CS Rb I absorption lines in several of these extreme O-rich AGB stars (see Papers I and II). Very recently, we have explored for the first time the $\mathrm{CS}$ effects on the $\mathrm{Rb}$ and $\mathrm{Zr}$ abundances derived in extreme O-rich AGB stars [5].

\section{Abundance analysis using dynamical models}

A modified version of the spectral synthesis code Turbospectrum [10] was used to deal with extended atmosphere models and velocity fields [5]. Our dynamical models are constructed from 


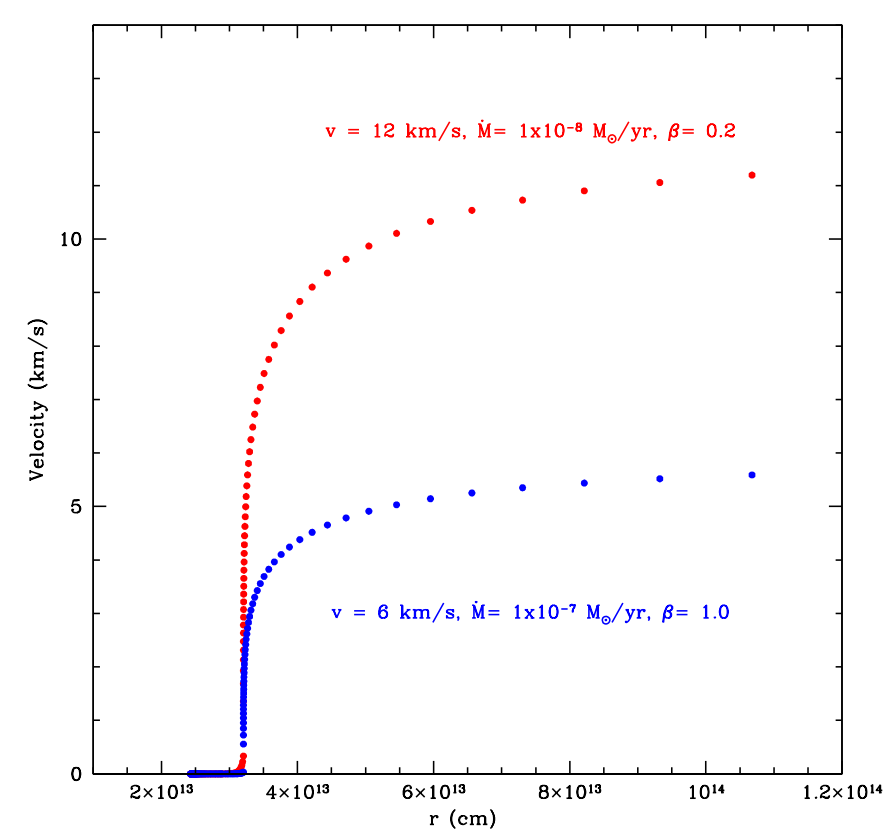

Figure 1: Velocity law (velocity vs. distance from the star) in two of our AGB wind models. Both models are based on the MARCS hydrostatic model with $\mathrm{T}_{\text {eff }}=3500 \mathrm{~K}, \log g=-0.5$, and solar chemical composition. The red model is computed with velocity at infinite of $12 \mathrm{kms}^{-1}$, mass-loss rate of $10^{-8} \mathrm{M}_{\text {sun }} \mathrm{yr}^{-1}$, and $\beta$ exponent of 0.2 . The blue model has a velocity at infinite of $6 \mathrm{kms}^{-1}$, mass-loss rate of $10^{-7} \mathrm{M}_{\text {sun }} \mathrm{yr}^{-1}$, and $\beta$ exponent of 1.0 .
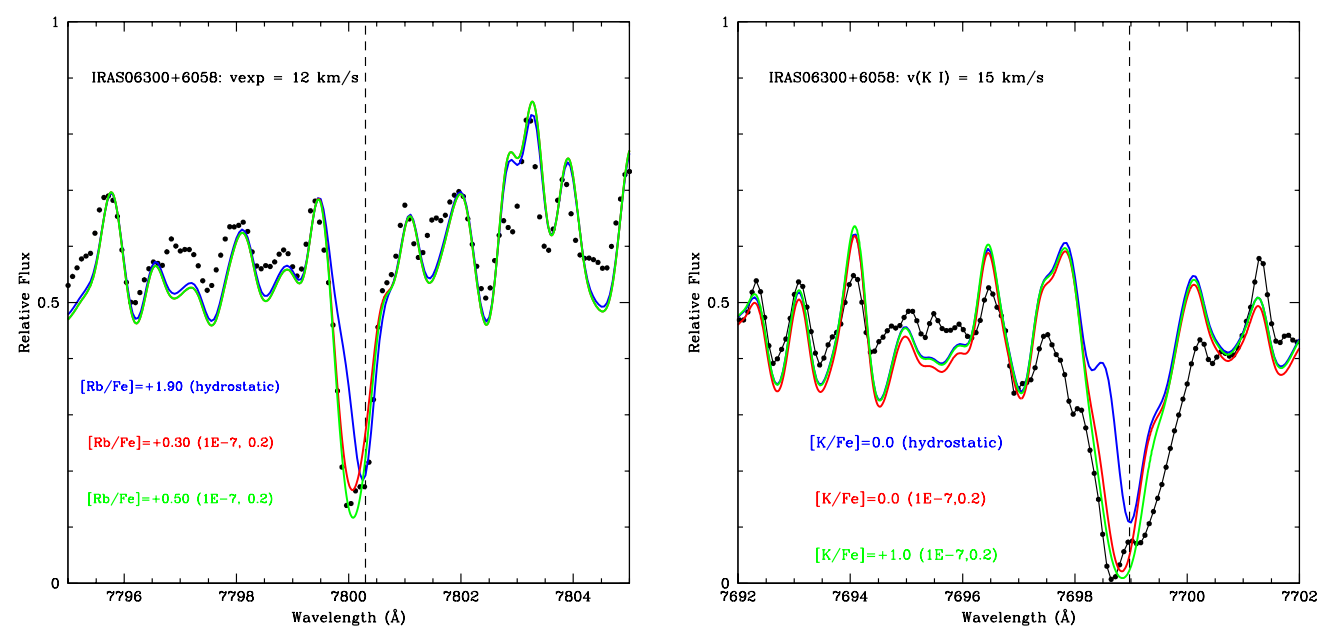

Figure 2: Rb I (left panel) and K I (right panel) line profiles obtained using dynamical models given by the $(\dot{M}, \beta)=\left(1 \times 10^{-7}, 0.2\right)$ pair for the star IRAS 06300+6058 [5]. The observations (black dots) and the hydrostatic models (blue lines) are also shown for comparison. The colours have similar meaning in the two panels but in the case of K I, all models were computed with a sligthly higher terminal velocity of 15 $\mathrm{kms}^{-1}$, as suggested by the blue-shift of the $\mathrm{K} \mathrm{I}$ line. We note that the red wing of the $\mathrm{K}$ I profile is not fully reproduced because of the presence of at least one interstellar component that is not seen in $\mathrm{Rb} \mathrm{I}$ [5]. 


\begin{tabular}{ccccc|ccc}
\hline \hline Star & $T_{\text {eff }}(\mathrm{K})$ & $\beta$ & $\dot{M}\left(\mathrm{M}_{\text {sun }} \mathrm{yr}^{-1}\right)$ & $v\left(\mathrm{~km} \mathrm{~s}^{-1}\right)$ & {$[\mathrm{Rb} / \mathrm{M}]_{\text {static }}$} & {$[\mathrm{Rb} / \mathrm{M}]_{\text {dyn }}$} & {$[\mathrm{Zr} / \mathrm{M}]_{\text {dyn }}$} \\
\hline \multicolumn{7}{c}{ Galactic stars } \\
\hline 05098 & 3000 & 1.0 & $1.0 \times 10^{-8}$ & 6 & $0.0 \pm 0.4$ & $0.0 \pm 0.4$ & $\leq 0.3 \pm 0.3$ \\
06300 & 3000 & 0.2 & $1.0 \times 10^{-7}$ & 12 & $1.9 \pm 0.4$ & $0.5 \pm 0.7$ & $\leq 0.1 \pm 0.3$ \\
18429 & 3000 & 1.0 & $1.0 \times 10^{-8}$ & 7 & $1.2 \pm 0.4$ & $1.0 \pm 0.4$ & $\leq 0.3 \pm 0.3$ \\
19059 & 3000 & 0.4 & $1.0 \times 10^{-7}$ & 13 & $2.4 \pm 0.4$ & $0.8 \pm 0.7$ & $\leq 0.3 \pm 0.3$ \\
\hline \multicolumn{7}{c}{ LMC star } \\
\hline 04498 & 3400 & 1.0 & $1.0 \times 10^{-7}$ & 13 & $3.3 \pm 0.4$ & $1.5 \pm 0.7$ & $\leq 0.3 \pm 0.3$ \\
\hline
\end{tabular}

Table 1: Atmosphere parameters and abundances derived using dynamical models vs. hydrostatic models.

the original MARCS hydrostatic atmosphere model structure [11], expanding the atmosphere radius by the inclusion of a wind out to $\sim 5$ stellar radii, with a radial velocity field in spherical symmetry. The stellar wind is computed under the assumptions of mass conservation and radiative thermal equilibrium, following a classical $\beta$-velocity law. Figure 1 shows examples of the $\beta$-velocity law adopted in our new dynamical models. In addition, in Figure 2, we show an example of the Rb I (left panel) and K I (right panel) line profiles obtained using our new dynamical models in comparison with the hydrostatic ones.

We note that we neglect line scattering in Turbospectrum but our complementary Monte Carlo simulations [5] (only taking into account photon scattering for the radiative transfer) confirm that our modified Turbospectrum code provides a reasonable approximation for the O-rich AGB stars studied here [5].

\section{Results}

The parameters of the dynamical atmosphere models providing the best fit to the observations and the derived $\mathrm{Rb}$ and $\mathrm{Zr}$ abundances $\left([\mathrm{Rb} / \mathrm{M}]_{d y n}\right.$ and $[\mathrm{Zr} / \mathrm{M}]_{d y n}$ ) are shown in Table 1 . The $\mathrm{Rb}$ abundances $\left([\mathrm{Rb} / \mathrm{M}]_{\text {static }}\right.$ ) from hydrostatic models are also shown for comparison. In Figure 3, we display the observed Rb I line profiles in our O-rich AGB sample (black dots) together with the best synthetic spectra as obtained from the new dynamical models (red lines) versus the static ones (blue lines); the best fits to the molecular $\mathrm{ZrO}$ bands are also shown for comparison.

The van Raai et al. standard nucleosynthesis models [3] for intermediate-mass AGB stars show that the predicted $\mathrm{Rb}$ abundances range from $[\mathrm{Rb} / \mathrm{M}] \sim 0.0$ up to $1.44 \mathrm{dex}$, depending on the progenitor mass and metallicity [3]; the predicted $\mathrm{Rb}$ production increases with increasing stellar mass and decreasing metallicity. Maximum $[\mathrm{Rb} / \mathrm{M}]$ overabundances of 1.04 and 1.44 are found for a solar metallicity $6.5 \mathrm{M}_{\text {sun }}$ star and for a LMC metallicity $6 \mathrm{M}_{\text {sun }}$ star, respectively [3]. More recently, Karakas et al. have delayed the beginning of the superwind phase in solar metallicity nucleosynthesis models of massive AGB stars [4]. These models produce more $\mathrm{Rb}$ than in the standard van Raai et al. models [3] because the star experiences more thermal pulses before the superwind phase at the very end of the AGB. The maximum $\mathrm{Rb}$ production $([\mathrm{Rb} / \mathrm{M}]=1.34 \mathrm{dex})$ is predicted to occur for the $6 \mathrm{M}_{\text {sun }}$ case [4]. 

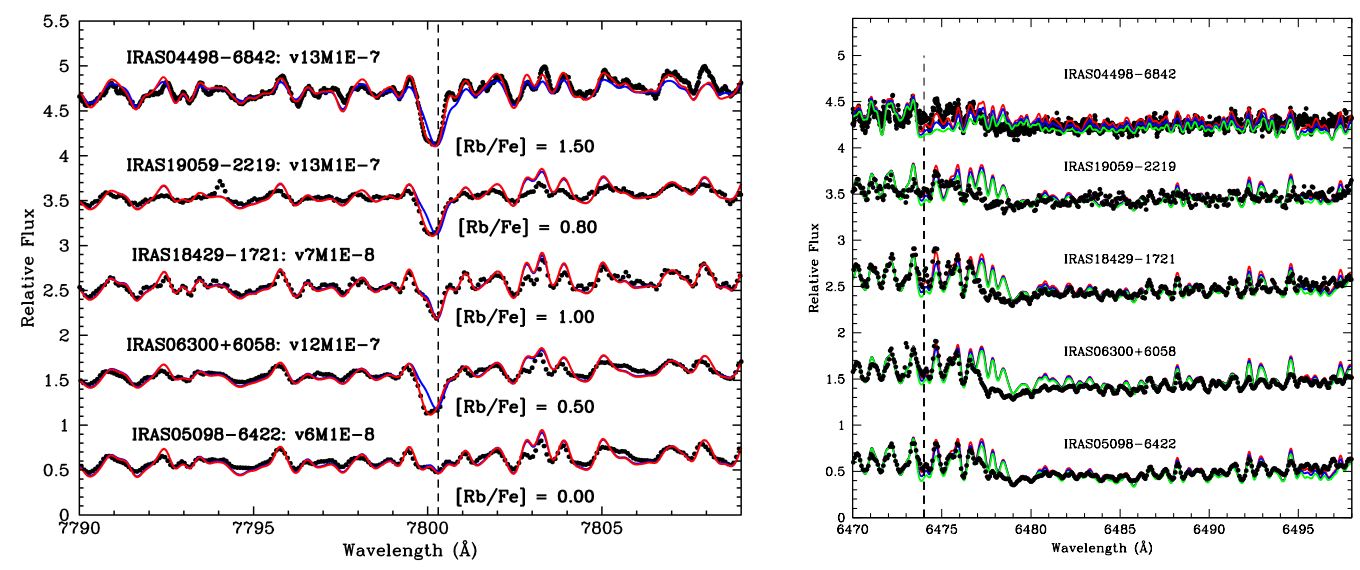

Figure 3: $\mathrm{Rb}$ (left panel) and $\mathrm{Zr}$ (right panel) abundances derived in the sample stars using dynamical models. Dynamical models providing the best fits to the observations (black dots) are indicated by a red line. Left panel: hydrostatic models are also shown for comparison (blue lines). The expansion velocity and the mass-loss rate adopted in the models also are indicated for each star. Right panel: synthetic spectra obtained for $\mathrm{Zr}$ abundances shifted +0.25 dex (in blue) and +0.50 dex (in green) from the adopted values are also shown.

By considering the error bars in the spectroscopic analysis (Table 1) and the theoretical uncertainties [3,4], the Rb abundances are now in fair agreement with the massive AGB nucleosynthesis models, both standard and with delayed superwinds. The nearly-solar derived $\mathrm{Zr}$ abundances in IRAS 05098+6422, IRAS 06300+6058, and IRAS 19059-2219 (Table 1) translate into [Rb/Zr] ratios of $-0.3,0.4$, and 0.5 , respectively, which agree quite well with the theoretical predictions $(-0.2$ $<[\mathrm{Rb} / \mathrm{Zr}]<0.6)$. However, the $[\mathrm{Rb} / \mathrm{Zr}]$ ratios in IRAS 18429-1721 (0.7) and IRAS 04498-6842 (1.2) are still higher than predicted. As already pointed out in the literature [3], a possible solution to this observational problem is that gaseous $\mathrm{Zr}$ condensates into dust grains, producing the apparent $\mathrm{Zr}$ underabundance.

\section{Conclusion}

In summary, the $\mathrm{Rb}$ abundances and $[\mathrm{Rb} / \mathrm{Zr}]$ ratios derived here significantly resolve the problem of the present mismatch between the observations of massive (4-8 $\mathrm{M}_{\text {sun }}$ ) Rb-rich AGB stars and the theoretical predictions. In the near future, we plan to carry out a chemical analysis based on these new dynamical models for all the Rb-rich AGB stars already studied in Papers I and II $[1,2]$. This undoubtedly will help us to constrain the actual nucleosynthesis models for the more massive AGB stars.

\section{References}

[1] D. A. García-Hernández et al., Rubidium-Rich Asymptotic Giant Branch Stars, Science, 314, (1751) 2006 (Paper I)

[2] D. A. García-Hernández et al., Rb-Rich Asymptotic Giant Branch Stars in the Magellanic Clouds, ApJ, 705, (L31) 2009 (Paper II) 
[3] M. A. van Raai et al., Rubidium, zirconium, and lithium production in intermediate-mass asymptotic giant branch stars, A\&A, 540, (A44) 2012

[4] A. I. Karakas et al., Heavy Element Nucleosynthesis in the Brightest Galactic Asymptotic Giant Branch Stars, ApJ, 751, (8) 2012

[5] O. Zamora et al., Circumstellar effects on the Rb abundances in O-rich AGB stars, A\&A, 564 (L4) 2014

[6] C. Abia et al.,The 85Kr s-Process Branching and the Mass of Carbon Stars, ApJ, 559 (1117) 2001

[7] D. L. Lambert et al., The Chemical Composition of Red Giants. IV. The Neutron Density at the s-Process Site, ApJ, 450, (302) 1995

[8] D. A. García-Hernández et al., Lithium and zirconium abundances in massive Galactic O-rich AGB stars, A\&A, 462, (711) 2007

[9] J. M. Trigo-Rodríguez et al. The role of massive AGB stars in the early solar system composition, $M \& P S, 44$ (627) 2009

[10] Alvarez, R. \& Plez, B., Near-infrared narrow-band photometry of M-giant and Mira stars: models meet observations, A\&A, 330, (1109) 1998

[11] B. Gustafsson et al., A grid of MARCS model atmospheres for late-type stars. I. Methods and general properties, A\&A, 486, (951) 2008 